\title{
ON THE TAILS OF THE EXPONENTIAL SERIES
}

\author{
C. YALÇIN YILDIRIM
}

\begin{abstract}
A relation between the zeros of the partial sums and the zeros of the corresponding tails of the Maclaurin series for $e^{z}$ is established. This allows an asymptotic estimation of a quantity which came up in the theory of the Riemann zeta-function. Some new properties of the tails of $e^{z}$ are also provided.
\end{abstract}

1. Introduction. We were led to this study of the exponential series from some mean-value estimates pertaining to the derivatives of the Riemann zeta-function ([2], [11]). It is well known that $\zeta(s)$ satisfies the functional equation

$$
\zeta(1-s)=\chi(1-s) \zeta(s) ; \quad \chi(1-s)=\pi^{\frac{1}{2}-s} \frac{\Gamma\left(\frac{s}{2}\right)}{\Gamma\left(\frac{1-s}{2}\right)} .
$$

In their work on the zeros $\rho_{k}$ of $\zeta^{(k)}(s)$ Conrey and Ghosh [2], assuming the Riemann Hypothesis, proved that

$$
\sum_{0<\Im \rho_{k} \leq T} \chi\left(\rho_{k}\right) \sim \alpha_{k} \frac{T}{2 \pi}
$$

as $T \rightarrow \infty$ (the number of $\rho_{k}$ with $0<\Im \rho_{k}<T$ is $\sim \frac{1}{2 \pi} T \log T$ ). Here

$$
\alpha_{k}:=k+1-\sum_{j=1}^{k} e^{-\nu_{j}}
$$

and $\nu_{j}=\nu_{j}(k)(j=1,2, \ldots, k)$ are the (distinct) roots of

$$
P_{k}(z):=\sum_{r=0}^{k} \frac{z^{r}}{r !}
$$

the $k$-th partial sum of the Maclaurin series for $e^{z}$. Conrey and Ghosh [3] also showed that for sufficiently large $k$

$$
\left|\alpha_{k}\right| \leq \dot{e}-\beta k
$$

with $0<\beta<1-\log 2$. Most of the numbers $e^{-\nu_{j}}$ are in fact exponentially large as functions of $k$, so it is indeed striking that $\alpha_{k}$ turns out to be exponentially small. Note that by Lindemann's theorem $\alpha_{k} \neq 0$.

Upon further scrutiny of the exponential series [12] the stronger estimate

$$
\alpha_{k} \ll_{m} k^{-m} e^{-k}
$$

Received by the editors July 9, 1992; revised March 4, 1993.

AMS subject classification: Primary: 33B10, 30C15; secondary: 30B10.

(C) Canadian Mathematical Society, 1994. 
for any fixed $m>0$, was reached [13]. Two ingredients were employed to obtain this result. One was bounds on the coefficients of the reciprocals of normalized tails of $e^{z}$. These bounds were found by determining the values assumed by the normalized tails on the unit disk and then applying Cauchy's estimate. The other was-and the exponential smallness of $\alpha_{k}$ really hinged on this-the fact that $s_{-2}=\cdots=s_{-k}=0$, where $s_{r}\left(=s_{r}(k)\right)=\sum_{j=1}^{k} \nu_{j}^{r}$. This also characterizes the partial sums of $e^{z}$ : All $k$-tuples $\left(\nu_{1}, \ldots, \nu_{k}\right)$ with the property $s_{-2}=\cdots=s_{-k}=0$ are formed by the roots of $P_{k}(z)$ (Sós and Turán [9]). (Another characterization, by Buckholtz [1], expresses that in a certain sense the zeros of partial sums of the exponential series have larger moduli than those of the partial sums of any other power series.)

In this paper we find the asymptotic value of $\alpha_{k}$ as $k \rightarrow \infty$. First it is found by direct estimation that

$$
\frac{1}{2 \pi i} \int_{|z|=R} \frac{z^{k}}{k ! P_{k}(z) Q_{k}(z)} d z=0,
$$

where

$$
Q_{k}(z)=e^{z}-P_{k}(z),
$$

as $R \rightarrow \infty$ through a sequence which avoids the poles. Then, by means of the residue theorem, a connection is established between the zeros of $P_{k}(z)\left(\nu_{j}(k), j=1, \ldots, k\right)$ and those of $Q_{k}(z)\left(\mu_{l}(k), l=1,2, \ldots\right)$.

THEOREM 1. For every $k \geq 2$

$$
\sum_{j=1}^{k} e^{-\nu_{j}}-\sum_{l=1}^{\infty} e^{-\mu_{l}}=k+1 .
$$

From some knowledge on the location of $\mu_{l}$ 's it will become clear that

$$
\sum_{l=1}^{\infty} e^{-\mu_{l}} \sim 2 \Re e^{-\mu_{1}}
$$

( $\mu_{1}$ denotes the zero of $Q_{k}(z)$ with the least modulus), and this furnishes the asymptotic formula for $\alpha_{k}$.

COROLlary 1. As $k \rightarrow \infty$,

$$
\alpha_{k} \sim-2 \Re e^{-(k+1)+\sqrt{2} \varrho_{1} \sqrt{k+1}+\frac{1-2 e_{1}^{2}}{3}}
$$

where $\varrho_{1}$ is the zero of $\operatorname{erfc}(z)$ closest to the origin,

$$
\varrho_{1} \approx-1.35+1.99 i
$$

$\left(\operatorname{erfc}(z)=\frac{2}{\sqrt{\pi}} \int_{z}^{\infty} e^{-t^{2}} d t\right.$ is the complementary error function $)$.

In what follows we shall recount some facts concerning the partial sums and tails of $e^{z}$ from the literature. Some further results on the tails will be demonstrated in $\S 3$. 
2. Outline of background knowledge. The Eneström-Kakeya theorem states that the modulus of any zero of $a_{0}+a_{1} x+\cdots+a_{k} x^{k}, a_{i} \in \mathbb{R}^{+}$, can be at most $\max \left(\frac{a_{0}}{a_{1}}, \frac{a_{1}}{a_{2}}, \ldots, \frac{a_{k-1}}{a_{k}}\right)$. Hence

$$
\left|\nu_{j}\right| \leq k
$$

It was first shown by Szegö [10] that the numbers $\frac{\nu_{j}}{k}$ cluster around the simple closed curve $\Gamma=\left\{z:\left|z e^{1-z}\right|=1,|z| \leq 1\right\}$ as $k \rightarrow \infty$, and conversely each point of the Szegö curve is a limit point of the normalized zeros. Moreover, as $k \rightarrow \infty$, the proportion of the normalized zeros which cluster along a given $\operatorname{arc}$ of $\Gamma$ is asymptotic to $\frac{1}{2 \pi} \Delta \arg z e^{1-z}$ as $z$ moves on the arc. In particular the proportion of the zeros with negative real parts tends to $\frac{1}{2}+\frac{1}{e \pi}$ as $k \rightarrow \infty$. Buckholtz [1] added that $\frac{\nu_{j}}{k}$ always lies in the exterior of $\Gamma$ within a distance of $\frac{2 e}{\sqrt{k}}$ from $\Gamma$.

It is well-suited for our purpose to present a sketch of how such results concerning the zeros of the partial sums and the tails were derived by Dieudonne [4]. To see the pattern involved in the distribution of the zeros of $P_{k}(z)$ and $Q_{k}(z)$ it is convenient to work with the normalized tails, $f_{n}$, defined by writing

$$
Q_{k}(z)=\frac{z^{k+1}}{(k+1) !} f_{k+1}\left(\frac{z}{k+1}\right)
$$

The power series for $f_{n}$ is

$$
f_{n}(z)=1+\frac{z}{\left(1+\frac{1}{n}\right)}+\frac{z^{2}}{\left(1+\frac{1}{n}\right)\left(1+\frac{2}{n}\right)}+\cdots+\frac{z^{p}}{\left(1+\frac{1}{n}\right)\left(1+\frac{2}{n}\right) \cdots\left(1+\frac{p}{n}\right)}+\cdots
$$

We set $n=k+1$ and the solutions of

$$
f_{n}(z)=\frac{n ! e^{n z}}{(n z)^{n}}
$$

are $\frac{\nu_{j}}{n}$. By virtue of (5) we need to consider only $|z| \leq 1$. Let $(D)$ be the domain obtained from the unit disk by erasing all points of distance $<r$ to $1, r$ being an arbitrarily small but fixed positive number. First it is shown that in $(D)$

$$
f_{n}(z)=\frac{1}{1-z}\left(1+\lambda_{n}(z)\right)
$$

where $\lambda_{n}(z) \rightarrow 0$ uniformly as $n \rightarrow \infty$. Using (9) and Stirling's formula in (8) gives

$$
\sqrt{2 \pi n}\left(\frac{e^{z-1}}{z}\right)^{n}=\frac{1}{1-z}\left(1+\tau_{n}(z)\right)
$$

where $\tau_{n}(z) \rightarrow 0$ uniformly in $(D)$ as $n \rightarrow \infty$. Taking $n$-th roots decomposes (10) into $n$ equations $(k=0,1, \ldots, n-1)$

$$
\frac{e^{z-1}}{z}=\left(\frac{1+\tau_{n}(z)}{\sqrt{2 \pi n}(1-z)}\right)^{\frac{1}{n}} e^{\frac{2 \pi k i}{n}}
$$


The equations (11) may be compared with

$$
\frac{e^{z-1}}{z}=e^{\frac{2 \pi k i}{n}}
$$

with solutions on $\Gamma$ to see the distribution of the zeros of the partial sums. Of course the immediate neighbourhood of 1 , and the possible zeros there, are left in the dark by this analysis.

To look for the zeros of the tails it is first shown that $\frac{(n-1) ! P_{n-1}(n z)}{(n z)^{n-1}}$ tends to $\frac{1}{1-\frac{1}{2}}$ as $n \rightarrow \infty$, uniformly in $\left(D^{\prime}\right)$, the domain formed by removing from $|z| \geq 1$ all points of distance $<r$ to 1 . Hence, using Stirling's formula, $f_{n}(z)=0$ may be expressed as

$$
\sqrt{2 \pi n}\left(\frac{e^{z-1}}{z}\right)^{n}=\frac{1}{z-1}\left(1+\eta_{n}(z)\right)
$$

where $\eta_{n}(z) \rightarrow 0$ uniformly in $\left(D^{\prime}\right)$ as $n \rightarrow \infty$. The last equation differs from (10) only in the sign of the right-hand side, and its solutions are close to those of (12) on the curve $\Gamma^{\prime}=\left\{z:\left|z e^{1-z}\right|=1,|z| \geq 1\right\}$. There are two branches of $\Gamma^{\prime}$, symmetrical with respect to the positive real axis. At large abscissae the branch in the upper half-plane behaves like $y=e^{x-1}$. Each of the equations (12) has an infinite number of solutions on $\Gamma^{\prime}$. If $z_{p}$ and $z_{p+1}$ denote two consecutive roots of (12) with the same $k$, then $\Im\left(z_{p+1}-z_{p}\right) \rightarrow 2 \pi$ as $p \rightarrow \infty$. If $z_{p}^{(q)}$ and $z_{p}^{\left(q^{\prime}\right)}$ are two roots of (12) corresponding respectively to the values $q$ and $q^{\prime}$ of $k$ and situated consecutively on $\Gamma^{\prime}$, then $\Im\left(z_{p}^{(q)}-z_{p}^{\left(q^{\prime}\right)}\right) \rightarrow \frac{2\left(q-q^{\prime}\right) \pi}{n}$ as $p \rightarrow \infty$. Again the neighbourhood of 1 has been unexplored. Dieudonné concludes by remarking that the zeros of the partial sums $P_{k}(n z)$ and the tails $f_{n}(z)$ are situated in a complementary fashion, together they tend to the curve $\Gamma \cup \Gamma^{\prime}=\left\{z:\left|z e^{1-z}\right|=1\right\}$ as $n \rightarrow \infty$.

We now give some lemmas the first two of which follow directly from the definition of $f_{n}(z)$.

LEMMA 1. For every positive integer $n$ and for all $z \in \mathbb{C}$

$$
f_{n}(z)(1-z)=1-\frac{z f_{n}^{\prime}(z)}{n} \text {. }
$$

LEMMA 2. For every positive integer $n$ and for all $z \in \mathbb{C}$

$$
z f_{n+1}(z)=f_{n}\left(\frac{n+1}{n} z\right)-1 \text {. }
$$

LEMMA 3. The zeros of $f_{n}(z)$ are in the region $|z|>1,\left|z e^{1-z}\right| \geq 1$.

PROOF. First we show that $f_{n}(z)$ has no zeros on the unit disk. By Lemma 1 if $f_{n}(w)=0$, then $w f_{n}^{\prime}(w)=n$. So, if $|w| \leq 1$, then $\left|f_{n}^{\prime}(w)\right| \geq n$. But on the unit disk the maximum of $f_{n}^{\prime}(z)$ is $f_{n}^{\prime}(1)=n$ and $f_{n}(1) \neq 0$. Now suppose $|z|>1$ and $\left|z e^{1-z}\right| \leq 1$. Then

$$
\left|1-\frac{(n z)^{n}}{n !} e^{-n z} f_{n}(z)\right|=\left|\left(z e^{1-z}\right)^{n} e^{-n} \sum_{p=0}^{n-1} \frac{n^{p} z^{p-n}}{p !}\right|<e^{-n} \sum_{p=0}^{n-1} \frac{n^{p}}{p !}<1,
$$


so $f_{n}$ cannot vanish.

The next is a result due to Buckholtz [1] which can be proved by employing the Cauchy inequality for derivatives in conjunction with Lemma 1.

LEMMA 4 ([1]). The points where $f_{n}(z)=1$ are situated within a distance $\frac{2 e}{\sqrt{n}}$ of $\Gamma^{\prime}$.

Now we can elicit more information about where $f_{n}$ vanishes. By Lemma 3, all such points lie to the left of $\Gamma^{\prime}$. First consider the zeros with large moduli. If $f_{n}(\omega)=0$ and $\omega$ is large, then by Lemmas 2 and $4, d\left(\omega, \Gamma^{\prime}\right) \ll \frac{\log |\Im \omega|}{n} \ll \frac{\Re \omega}{n}$. Clearly $\arg \omega \rightarrow \frac{\pi}{2}^{-}$. Lemmas 2, 3 and 4 imply further that, for sufficiently large $n$, the zeros of $f_{n}$ with smallest moduli must also be those closest to 1 . Lemma 4 suggests examining the possibility $f_{n}\left(1+\frac{s}{\sqrt{n}}\right)=0$, where $s$ is in a fixed compact set. An alternative expression for $f_{n}$ appropriate for this purpose is ([7])

$$
\begin{gathered}
f_{n}(z)=1+\frac{n !}{(n z)^{n}}\left(e^{n z}-\frac{1}{n !} \int_{0}^{\infty} e^{-t}(t+n z)^{n} d t\right) \quad \text { i.e. } \\
f_{n}\left(1+\frac{s}{\sqrt{n}}\right)=1+\frac{e^{s \sqrt{n}}}{\left(1+\frac{s}{\sqrt{n}}\right)^{n}}\left(\frac{n ! e^{n}}{n^{n}}-\sqrt{n} \int_{s}^{\infty}\left(1+\frac{\zeta}{\sqrt{n}}\right)^{n} e^{-\zeta \sqrt{n}} d \zeta\right)
\end{gathered}
$$

upon putting $t=\sqrt{n}(\zeta-s)$, where the path of integration is the horizontal line from $s$ to the right to $\infty$. As $n \rightarrow \infty, \frac{e^{s \sqrt{n}}}{\left(1+\frac{s}{\sqrt{n}}\right)^{n}}$ converges to $e^{\frac{s^{2}}{2}}$ uniformly on every compact set in the $s$-plane. By the dominated convergence theorem, $\int_{s}^{\infty}\left(1+\frac{\zeta}{\sqrt{n}}\right)^{n} e^{-\zeta \sqrt{n}} d \zeta$ converges uniformly to $\int_{s}^{\infty} e^{-\frac{\zeta^{2}}{2}} d \zeta$ on any compact set in $\Im s \geq 0$. If $f_{n}\left(1+\frac{s_{n}}{\sqrt{n}}\right)=0$, then from (14) it is seen that, by virtue of Hurwitz's theorem, as $n \rightarrow \infty, s_{n}$ tends to a limit $s_{l}$ such that $\frac{1}{\sqrt{2 \pi}} \int_{s_{l}}^{\infty} e^{-\frac{\zeta^{2}}{2}} d \zeta=1$. Hence erfc $\left(\frac{-s_{l}}{\sqrt{2}}\right)=0$. The first hundred zeros of $\operatorname{erfc}(z)$ have been calculated by Fettis-Caslin-Cramer [5] (the first three pairs are approximately $-1.35 \pm 1.99 i,-2.18 \pm 2.89 i,-2.78 \pm 3.24 i)$. In particular, as $n \rightarrow \infty$, the zeros of $f_{n}(z)$ with the least modulus are $1+\frac{\sqrt{2}(1.35 \cdots \pm i 1.99 \cdots)+o(1)}{\sqrt{n}}$.

3. The zeros of the tails of $e^{z}$. We shall presently return to the zeros with the least modulus after a little digression. Let the zeros $\omega_{l}$ of $f_{n}$ (which are in conjugate pairs) be numbered as $\left|\omega_{1}\right|=\left|\omega_{2}\right|<\left|\omega_{3}\right|=\left|\omega_{4}\right|<\cdots, \Im \omega_{2 l+1}>0$. By inverting (7), write

$$
\frac{1}{f_{n}(z)}=\sum_{p=0}^{\infty} d_{p} z^{p},
$$

the inversion being valid for $|z|<\left|\omega_{1}\right|$.

THEOREM 2. The normalized tail of $e^{z}, f_{n}(z)$, possesses the infinite product representation (uniformly convergent on compact subsets of $\mathbb{C}$ )

$$
f_{n}(z)=e^{\frac{n}{n+1} z} \prod_{l}\left(1-\frac{z}{\omega_{l}}\right) e^{\frac{z}{\omega_{l}}}
$$


PROOF. Clearly $f_{n}(z)$ is an entire function of order 1, with Hadamard factorization

$$
f_{n}(z)=e^{a z} \prod_{l}\left(1-\frac{z}{\omega_{l}}\right) e^{\frac{z}{\omega_{l}}} .
$$

By logarithmic differentiation, when $|z|<\left|\omega_{1}\right|$,

$$
\frac{f_{n}^{\prime}}{f_{n}}(z)=a-\sum_{p=1}^{\infty}\left(\sum_{l} \frac{1}{\omega_{l}^{p+1}}\right) z^{p} .
$$

(Note that the exponent of convergence of $f_{n}(z)$ is 1 , therefore $\sum_{l} \frac{1}{\left|\omega_{l}\right|^{p}}$ is convergent if $p \geq 2$. Dieudonné's results related in $\S 2$ reveal that $\sum_{l} \frac{1}{\left|\omega_{l}\right|}$ is divergent. However, by Lindelöf's theorem (see e.g. [6], p. 20), $\sum_{\left|\omega_{l}\right| \leq r} \frac{1}{\omega_{l}}$ is bounded as $r \rightarrow \infty$.) On the other hand from Lemma 1 and (15) we also have

$$
\frac{f_{n}^{\prime}}{f_{n}}(z)=\frac{n}{z}\left(\frac{1}{f_{n}(z)}+z-1\right)=\frac{n}{n+1}-n \sum_{p=1}^{\infty} d_{p+1} z^{p} .
$$

Comparing alike coefficients in (16) and (17) shows that $a=\frac{n}{n+1}$, and also

COROLLARY 2. $d_{p}=\frac{1}{n} \sum_{l} \frac{1}{\omega_{l}^{p}} ;(p \geq 2)$.

Corollary 3. $\left|d_{p}\right| \ll\left|\omega_{1}\right|^{-p} ;(p \geq 2)$.

PROOF. Observe that for all sufficiently large $n, \Re \omega_{l}>1$. From the way the zeros are located we infer that $\Re\left(\omega_{l}^{5}\right)>1$ except for some zeros at a distance $O(1)$ from 1 . The number of such exceptional zeros must be $O(n)$ by $\S 2$. Then, for $p \geq 10$, we have by Corollary 2

$$
\begin{aligned}
n\left|d_{p}\right| & \leq \sum_{l} \frac{1}{\left|\omega_{l}\right|^{p}} \\
& \leq \frac{1}{\left|\omega_{1}\right|^{p-10}} \sum_{l} \frac{1}{\left|\omega_{l}\right|^{10}} \\
& \leq \frac{1}{\left|\omega_{1}\right|^{p-10}}\left(\sum_{l} \frac{\Re\left(\omega_{l}^{5}\right)}{\left|\omega_{l}\right|^{10}}+O(n)\right) \\
& =\frac{1}{\left|\omega_{1}\right|^{p-10}}\left(\sum_{l} \frac{1}{\omega_{l}^{5}}+O(n)\right) \\
& =\frac{1}{\left|\omega_{1}\right|^{p-10}}\left(n d_{5}+O(n)\right),
\end{aligned}
$$

and Corollary 3 is proved. We note in passing that Cauchy's estimate on the unit disk implies $d_{p} \ll \frac{1}{\sqrt{n}}$ for $p \geq 2$.

We now return to the problem of locating precisely the least-moduli zeros of $f_{n}$ in terms of the zeros of $\operatorname{erfc}(z)$. The asymptotic expansion of $f_{n}(z)$ by Soni and Soni [8] lends itself to this end. Let

$$
\begin{gathered}
z=e^{\alpha} \\
b=\alpha\left\{2\left(e^{\alpha}-1-\alpha\right) / \alpha^{2}\right\}^{\frac{1}{2}},
\end{gathered}
$$


where $z=1$ corresponds to $\alpha=0$ and the principal branch of the square-root function is taken. Then $b=b(z)$ satisfies

$$
e^{-\frac{b^{2}}{2}}=z e^{1-z}
$$

Also put

$$
\begin{aligned}
H_{n}(b) & :=\int_{0}^{\infty} e^{-n\left(\frac{w^{2}}{2}-b w\right)} d w \\
& =\sqrt{\frac{\pi}{2 n}} e^{\frac{n b^{2}}{2}} \operatorname{erfc}\left(-\sqrt{\frac{n}{2}} b\right) .
\end{aligned}
$$

LEMMA 5 ([8]). Let $\Omega$ be a compact set in $\mathbb{C}$ with the cut, $|\arg z|<\pi,|z|>0$. As $n \rightarrow \infty$, uniformly for all $z \in \Omega$,

$$
\begin{aligned}
f_{n}(z)=1 & +\left(n+\frac{1}{12}+\frac{1}{288 n}\right) H_{n}(b)-\frac{z}{z-1}+\frac{1}{b}+\frac{z}{n(z-1)^{3}} \\
& +\left(\frac{1}{12 b}-\frac{1}{b^{3}}\right) \frac{1}{n}+O\left(\frac{1}{n} \max \left(1, H_{n}(b)\right)\right) .
\end{aligned}
$$

Let $\varrho$ be a zero of $\operatorname{erfc}(z)$. Based on the discussion following Lemma 4 , for $f_{n}(\omega)=0$ we try

$$
\omega=1-\sqrt{\frac{2}{n}} \varrho+\frac{c}{n}+O\left(n^{-\frac{3}{2}}\right),
$$

where $c$ will be determined. Then

$$
b(\omega)=-\sqrt{\frac{2}{n} \varrho}+\frac{c-\frac{2}{3} \varrho^{2}}{n}+O\left(n^{-\frac{3}{2}}\right),
$$

and using the Taylor expansion (up to second order) of $\operatorname{erfc}(z)$ around $z=\varrho$ in (18)

$$
H_{n}(b)=\frac{c-\frac{2}{3} \varrho^{2}}{n}+O\left(n^{-\frac{3}{2}}\right)
$$

These are plugged in the formula of Lemma 5 to obtain $c=\frac{2 \varrho^{2}-1}{3}$, so that

LEMMA 6. If $\operatorname{erfc}(\varrho)=0$, $\varrho$ fixed, then as $n \rightarrow \infty$

$$
f_{n}\left(1-\sqrt{\frac{2}{n} \varrho}+\frac{2 \varrho^{2}-1}{3 n}+O\left(n^{-\frac{3}{2}}\right)\right)=0 .
$$

4. Proof of Theorem 1. By (6), the zeros of $Q_{k}(z)$ are at $n \omega_{l}=\mu_{l}$, say. From the discussion in $\S 2$ we know that $\left|\mu_{l+2}\right|-\left|\mu_{l}\right| \rightarrow 2 \pi$ as $l \rightarrow \infty$. So in order to have the contour of integration in (3) stay clear of the zeros of $Q_{k}(z)$ we choose $R_{l}=\frac{\left|\mu_{l}\right|+\left|\mu_{l+2}\right|}{2}$, and write $R=\left(R_{l}\right)_{l=1}^{\infty}$. Now we claim that if $k \geq 2$, then

$$
\lim _{l \rightarrow \infty} \frac{1}{2 \pi i} \int_{|z|=R_{l}} \frac{z^{k}}{k ! P_{k}(z) Q_{k}(z)} d z=0 .
$$


To see this we examine the values of the integrand on the semicircle $z=R e^{i \theta}, 0 \leq \theta \leq \pi$. As $R \rightarrow \infty$,

$$
\left|\frac{z^{k}}{k ! P_{k}(z)}\right|=1+O_{k}\left(\frac{1}{R}\right) .
$$

For $0<A_{1} \leq A \leq A_{2}$, where $A_{1}$ and $A_{2}$ are fixed, the equality $\left|e^{z}\right|=A\left|P_{k}(z)\right|$ is achieved at

$$
\theta=\arccos \left(\frac{k \log R-\log k !+\log A}{R}+O\left(\frac{1}{R^{2}}\right)\right) .
$$

Let $\phi_{1}$ and $\phi_{2}$ (resp.) be the angles (in the first quadrant) so determined for $A=\frac{1}{2}$ and $A=\frac{3}{2}$ (resp.). If $\theta \notin\left[\phi_{1}, \phi_{2}\right]$, then

$$
\left|Q_{k}(z)\right|=\left|e^{z}-P_{k}(z)\right|>\frac{R^{k}}{3 k !} .
$$

At the large-moduli zeros of $Q_{k}(z)$ we have roughly

$$
e^{\left|\mu_{l}\right| \cos \theta_{l}} e^{i\left|\mu_{l}\right| \sin \theta_{l}}=\frac{\left|\mu_{l}\right|^{k}}{k !} e^{i k \theta_{l}}
$$

Since $\sin \theta_{l} \rightarrow 1$, it is seen once again that $\left|\mu_{l+2}\right|-\left|\mu_{l}\right| \sim 2 \pi$. Moreover, using $R_{l}$ in place of $\left|\mu_{l}\right|$ renders the left-hand side of (22) diametrically opposite to what it is with $\left|\mu_{l}\right|$. Hence (21) holds for $\theta \in\left[\phi_{1}, \phi_{2}\right]$ too, provided that $R \in \mathcal{R}$. This proves (19).

The residues of the integrand at its poles are

$$
\begin{gathered}
\operatorname{Res}\left(\frac{z^{k}}{k ! P_{k}(z) Q_{k}(z)} ; \nu_{j}\right)=\frac{-1}{Q_{k}\left(\nu_{j}\right)}=-e^{-\nu_{j}}, \\
\operatorname{Res}\left(\frac{z^{k}}{k ! P_{k}(z) Q_{k}(z)} ; \mu_{l}\right)=\frac{1}{P_{k}\left(\mu_{l}\right)}=e^{-\mu_{l}}, \\
\operatorname{Res}\left(\frac{z^{k}}{k ! P_{k}(z) Q_{k}(z)} ; 0\right)=k+1 .
\end{gathered}
$$

Together with (19) this completes the proof of Theorem 1.

ACKNOWLEDGEMENTS. I am indebted to the referee for his/her suggestion of the present version of the results. Formerly I had deduced the same estimate as here (save for a constant factor) as an upper-bound for $\alpha_{k}$ from Corollary 3.

\section{REFERENCES}

1. J. D. Buckholtz, A characterization of the exponential series, Amer. Math. Monthly (2) 73(1966), 121-123.

2. J. B. Conrey and A. Ghosh, Zeros of derivatives of the Riemann zeta-function near the critical line, Analytic Number Theory, (ed. et al. Berndt), Progr. Math. 85, Birkhäuser Boston, 1990.

3. $\longrightarrow$ On the zeros of the Taylor polynomials associated with the exponential function, Amer. Math. Monthly 95(1988), 528-533.

4. J. Dieudonné, Sur les zéros des polynômes-sections de $e^{x}$, Bull. Sci. Math. 70(1935), 333-351.

5. H. E. Fettis, J. C. Caslin and K. R. Cramer, Complex zeros of the error function and of the complementary error function, Math. Comp. 27(1973), 401-404. 
6. P. Koosis, The Logarithmic Integral I, Cambridge, 1988.

7. D. J. Newman and T. J. Rivlin, The zeros of the partial sums of the exponential function, J. Approx. Theory 5(1972), 404-412; Correction J. Approx. Theory 16(1976), 299-300.

8. K. Soni and R. P. Soni, An approximation connected with the exponential function, Proc. Amer. Math. Soc. (4) 114(1992), 909-918.

9. V. T. Sós and P. Turán, On some new theorems in the theory of Diophantine approximations, Acta Math Hungar. 6(1955), 241-255.

10. G. Szegö, Über eine eigenschaft der Exponentialreihe, Sitzungsber. Berlin Math. Ges. 23(1924), 50-64.

11. C. Y. Yildırım, The mean value of $\left|\zeta\left(\frac{1}{2}+i t\right)\right|^{2}$ at the zeros of $Z^{(k)}(t)$, C. R. Math. Rep. Acad. Sci. Canada (4) XII(1990), 135-140.

12. - A sum over the zeros of partial sums of $e^{x}$, J. Ramanujan Math. Soc. 6(1991), 51-66.

13. On the zeros of the sections of the exponential function, Turk. J. Math. (3) 16(1992), 177-182.

Department of Mathematics

Bilkent University

Ankara 06533

Turkey 